\title{
Occupational moral injury and mental health: systematic review and meta- analysis
}

Victoria Williamson, Sharon A.M. Stevelink and Neil Greenberg

\section{Background}

Many people confront potentially morally injurious experiences (PMIES) in the course of their work which can violate deeply held moral values or beliefs, putting them at risk for psychological difficulties (e.g. post-traumatic stress disorder (PTSD), depression, etc.).

\section{Aims}

We aimed to assess the effect of moral injury on mental health outcomes.

\section{Method}

We conducted a systematic review and meta-analysis to assess the association between work-related PMIEs and mental health disorders. Studies were independently assessed for methodological quality and potential moderator variables, including participant age, gender and PMIE factors, were also examined.

\section{Results}

Thirteen studies were included, representing 6373 participants PMIEs accounted for $9.4 \%$ of the variance in PTSD, $5.2 \%$ of the variance in depression and $2.0 \%$ of the variance in suicidality. PMIES were associated with more symptoms of anxiety and behavioural problems (e.g. hostility), although this relationship was not consistently significant. Moderator analyses indicated that methodological factors (e.g. PMIE measurement tool), demographic characteristics and PMIE variables (e.g. military $v$. non-military context) did not affect the association between a PMIE and mental health outcomes.

\section{Conclusions}

Most studies examined occupational PMIEs in military samples and additional studies investigating the effect of PMIEs on civilians are needed. Given the limited number of high-quality studies available, only tentative conclusions about the association between exposure to PMIEs and mental health disorders can be made.

\section{Declaration of interest}

None.

\section{Keywords}

Moral injury; meta-analysis; systematic review; occupation; PTSD; depression; suicidality.

\section{Copyright and usage}

(c) The Royal College of Psychiatrists 2018.
Potentially morally injurious experiences (PMIEs), including 'perpetrating, failing to prevent, bearing witness to, or learning about acts that transgress deeply help moral beliefs and expectations' (Litz et al., ${ }^{1}$ p. 700), can result in significant psychological distress or moral injury. ${ }^{1}$ Certain occupational groups may be at risk of exposure to work-related morally injurious events, including first responders, journalists and armed forces personnel. Moral injury is often associated with strong moral emotions related to the event, including guilt, anger and disgust, ${ }^{2}$ and can lead to distress and psychological difficulties. For example, in combat veterans, PMIEs is significantly associated with post-traumatic stress disorder (PTSD), depression and suicidal ideation. ${ }^{2,3}$ However, the psychological effect of PMIEs on those in non-military employment remains unclear. Most studies have exclusively examined moral injury in US armed forces personnel. ${ }^{4-7}$ The few studies that exist indicate that those in non-military professions, such as police, can also experience moral injury after a PMIE. ${ }^{8,9}$ The aim of this review is to examine the mental health outcomes associated with occupational PMIEs. We also examined potential moderators of effects to determine whether these influenced the magnitude of the associations between PMIEs and mental health outcomes. Studies examining moral distress, which is similar to moral injury, in healthcare professionals have found that exposure can cause psychological distress and burnout. ${ }^{10,11}$ As this concept has been extensively reviewed in recent years (e.g. and Lamiani et al. ${ }^{10}$ and $\mathrm{Oh}$ and Gastmans ${ }^{11}$ ), it was not included in our study.

\section{Method}

\section{Search strategy}

We conducted a computer-based search in December 2016 of the following psychological and medical electronic literature databases:
Embase, PsychNet, Medline, PsycInfo, PILOTS, Google Scholar and Web of Science. The search terms were related to moral injury, mental health and occupation (see Supplementary Table 1 available at https://doi.org/10.1192/bjp.2018.55). In addition, key authors were contacted to request details of any further studies and reference sections of relevant review articles (e.g. Litz et al., ${ }^{1}$ Frankfurt and Frazier, ${ }^{3}$ and Dombo et al. ${ }^{12}$ ), book chapters and issues of journals (e.g. Journal of Traumatic Stress) were manually searched to identify any additional studies.

\section{Eligibility criteria}

Studies had to meet the following criteria to be considered for inclusion: a direct measure of exposure to PMIE incurred as a result of the participant's occupation, a standardised measure of mental health and statistical testing of the association between PMIEs and mental health. Measures of exposure to PMIEs were included if they asked about exposure to occupation-related perceived transgressions committed by the respondent and/or other individuals, or perceived betrayal by others, such as colleagues. ${ }^{1-3}$

Studies were excluded on the following grounds: (a) the article was a review that did not offer new data or only presented qualitative analysis, (b) single case studies, (c) studies not written in English or (d) studies examining moral distress in nursing and medical professionals.

Two authors (V.W. and S.A.M.S.) independently screened articles and extracted data. A Preferred Reporting Items for Systematic Reviews and Meta-Analyses flow chart (Fig. 1) delineates the review process. ${ }^{13}$ On two occasions, the same data were reported in more than one article. In such cases, results from the most comprehensive 


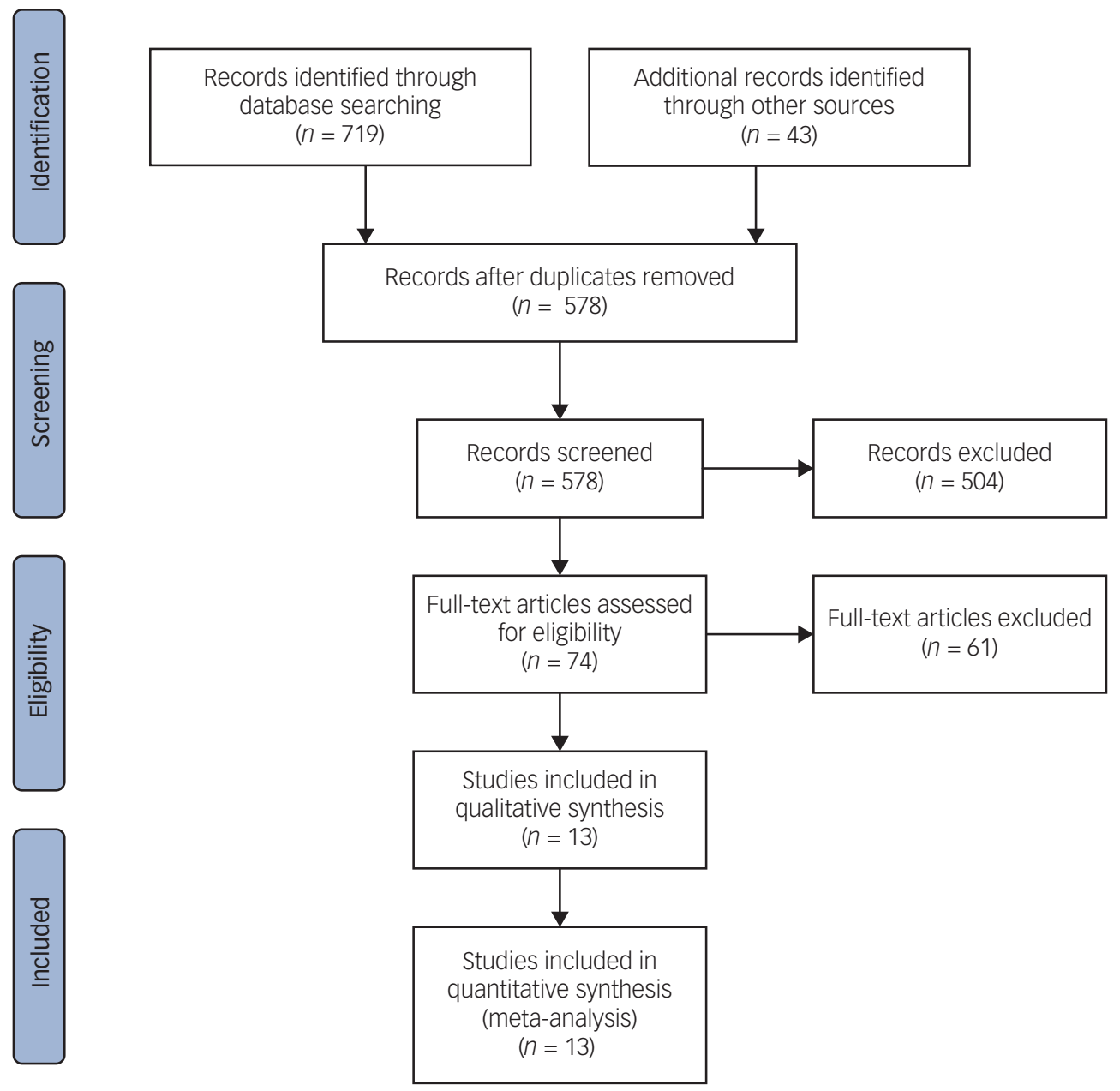

Fig. 1 Preferred Reporting Items for Systematic Reviews and Meta-Analyses flow diagram.

article were used. ${ }^{14}$ The final sample consisted of 13 studies that met the inclusion criteria.

\section{Data extraction}

The following data were extracted from each study, if available: (a) author name, (b) publication year, (c) study design, (d) study location, (e) type of PMIEs, (f) instrument used to assess the PMIE, (g) sample size, (h) gender distribution, (i) participant age, (j) assessment time points, (k) mental health disorder assessed, (l) mental health instruments/diagnostic criteria used, (m) time since PMIE, (n) findings and effect sizes and (o) any sources of bias or ethical issues. The data was extracted and assessed independently by two authors (V.W. and S.A.M.S.). Any discrepancies were checked and a consensus was successfully reached.

\section{Quality rating}

Two authors (V.W. and S.A.M.S.) independently assessed the methodological quality of all included studies by a seven-item checklist adapted from Ajetunmobi. ${ }^{15}$ The highest possible quality score was seven, indicative of a better-quality study, with zero as the lowest possible score. Adapted items on the checklist include an evaluation of whether the study design was evident and appropriate, if random sampling of study participants was used to ensure all members of the examined population had an equal chance of being selected into the sample and if the analytic methods used were well described and appropriate. Studies were scored depending on the extent to which the specific criteria were met (with yes being one and no being zero) and we calculated a summary score for each study by summing the total score across all items of the scale (see Table 1 and Supplementary Table 2). Agreement between authors was strong, with any disagreements resolved in a consensus meeting.

\section{Data synthesis}

The relationship between PMIEs and PTSD, depression and suicidal ideation was examined by meta-analytic methods. The relationship between PMIEs and anxiety and well-being (e.g. resilience, hostility, stress, positive affect and social adjustment) was examined descriptively, owing to insufficient data for meaningful statistical analysis $(k<4)$.

We conducted meta-analyses, using Rstudio (version 0.98.507) with the Metafor package. ${ }^{41}$ We used Pearson's product-moment correlation $(r)$ as the effect size because $r$ is more readily interpretable in comparison to other effect sizes ${ }^{42,43}$ and is easily computed from $t, F$ and $d$. We extracted effect size values for each association of interest within each study, with separate effect size values for each mental health disorder. Where necessary, correlation coefficients were calculated from other provided effect sizes (e.g. F) or obtained from study authors. Cohen's ${ }^{44}$ guidelines were used to interpret the effect sizes (small effect $r=0.10$, moderate effect $r=0.30$ and large effect $r=0.50$ ). Correlation coefficients were computed so that a positive coefficient reflected more severe mental health disorder 
Table 1 Included studies sample characteristics, methods of assessment and quality ratings

\begin{tabular}{|c|c|c|c|c|c|c|c|c|c|}
\hline Study & Location & Design & $\mathrm{N}$ & Males (\%) & $\begin{array}{l}\text { Age, mean } \\
\text { years (SD) }\end{array}$ & Exposure & Well-being measured & PMIE measurement & $\begin{array}{l}\text { Quality } \\
\text { rating }\end{array}$ \\
\hline Backholm and Idas ${ }^{8}$ & Norway & Cross-sectional & 371 & 59.6 & $36.03(10.09)$ & $\begin{array}{l}\text { Journalists covering } 2011 \text { Norway } \\
\text { terrorist attack }\end{array}$ & PTSD (IES-R) & Three-item questionnaire & 5 \\
\hline Bryan et al. ${ }^{4}$ a & USA & Cross-sectional & 151 & 63.8 & $34.12(8.41)$ & Afghanistan/Iraq deployment & $\begin{array}{l}\text { Suicidal ideation (BSSI), PTSD (PCL-M), depression } \\
\quad(\text { PHQ-9) }\end{array}$ & MIES & 5 \\
\hline Bryan et al. ${ }^{5}$ a & USA & Cross-sectional & 935 & 82.3 & $27.05(8.11)$ & Afghanistan/Iraq deployment & $\begin{array}{l}\text { Suicidal ideation (BSSI), PTSD (PCL-M), depression } \\
\text { (PHQ-9), anxiety (GAD-7), hostility (STAXI-2) }\end{array}$ & MIES & 6 \\
\hline Crane et al. ${ }^{16}$ & Australia & Cross-sectional & 540 & 35.8 & $41.06(11.53)$ & Veterinary practice & $\begin{array}{l}\text { Depression, stress and anxiety (DASS-21), resilience } \\
\text { (BRS) }\end{array}$ & Stressor checklist & 4 \\
\hline Currier et al. ${ }^{14 \mathrm{~b}}$ & USA & Cross-sectional & 213 & 88.0 & $28.47(5.87)$ & Afghanistan/Iraq deployment & $\begin{array}{l}\text { Suicidal ideation (SBQ-R), depression (PHQ-9), PTSD } \\
\quad \text { (PLC-C) }\end{array}$ & MIQ-M & 5 \\
\hline Currier et al. ${ }^{17}$ & El Salvador & Cross-sectional & 257 & 31.4 & $.02(13.12$ & $\mathrm{omm}$ & PTSD (LASC) & MIQ-T & 5 \\
\hline Dennis et al. ${ }^{18}$ & USA & Cross-sectional & 603 & 100.0 & $51.0(5.71)$ & Vietnam war & $\begin{array}{l}\text { Suicidal ideation (SBQ-R), PTSD (PLC-C), depression } \\
\text { (PHQ-9), hostility (CMHS) }\end{array}$ & VESI & 4 \\
\hline Ferrajão and Oliveira' ${ }^{19}$ & Portugal & Cross-sectional & 60 & 100.0 & A) & ortus & PTSD (IES-R), depression (BSI) & Interview & \\
\hline Komarovskaya et al..$^{9}$ & USA & Cross-sectional & 400 & 85.0 & $27.0(4.81)$ & $\begin{array}{l}\text { Killing/seriously injuring others during } \\
\text { police service }\end{array}$ & PTSD (MCS-CV), social adjustment (SAS-SR) & $\mathrm{ClHQ}$ & 4 \\
\hline Nash et al. $^{7}$ & USA & Longitudinal & 533 & N/A & $22.67(3.50)$ & Afghanistan/Iraq deployment & $\begin{array}{l}\text { PTSD (PCL), anxiety (BAI) depression (BDI-II), positive } \\
\text { affect (PANAS), social adjustment (ISEL) }\end{array}$ & MIES & 5 \\
\hline Ritov and Barnetz ${ }^{20}$ & Israel & Cross-sectional & 147 & 100.0 & 67) & Combat service in We & PTSD (unnamed) & Moral objection & 4 \\
\hline Tripp et al. 21a & ISA & Cross-sectional & 68 & 91.0 & 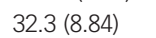 & Afghanistan//raq deployment & PTSD (CAPS), depress & & 3 \\
\hline Wilk et al. ${ }^{22}$ & USA & Cross-sectional & 2095 & 91.0 & N/A & Afghanistan/Iraq deployment & PTSD (PCL), hostility (unnamed) & BEB & 5 \\
\hline \multicolumn{10}{|c|}{ 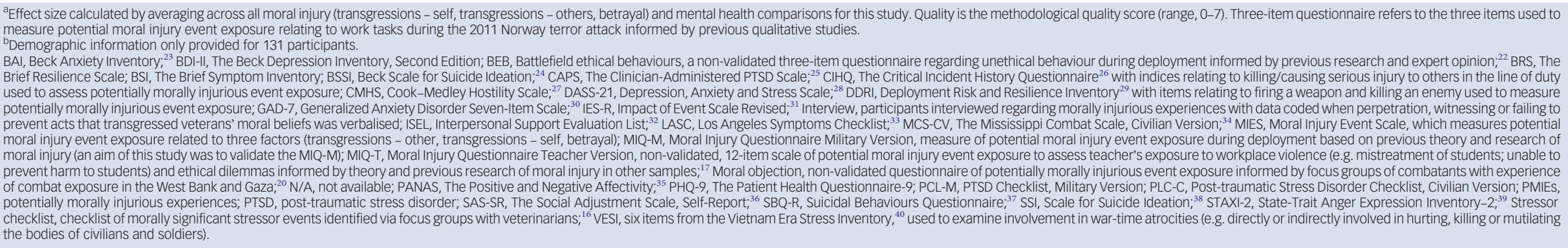 } \\
\hline
\end{tabular}


symptoms, and a negative coefficient reflected less severe mental health disorder symptoms.

Where data regarding the relationship between particular PMIEs (e.g. transgressions - self, transgressions - others, betrayal) and mental health disorder symptoms was reported, one effect size was generated for each study by averaging across all of the PMIEs and mental health comparisons for the study. ${ }^{4,5}$ Study effect sizes by event type can be found in Supplementary Table 3. If studies recruited two samples but administered a more complete battery of mental health assessments to one particular sample, the data from this sample was used in the analyses (e.g. Bryan et al. ${ }^{5}$ ).

We applied the Hedges-Olkin approach, ${ }^{45}$ using the Fisher transformed correlation coefficients with the results reported in Pearson's $r$ following a back conversion. We chose random-effects modelling with restricted maximum likelihood a priori, as this method allows the meta-analytic results to be generalised to a wider population of studies. ${ }^{45,46}$ To assess heterogeneity, or the presence of variation in the true effect sizes between studies, Cochran's $Q$ and $I^{2}$ statistic were used. Heterogeneity can be clinical (e.g. differences between patients), methodological (e.g. differences in study design) or statistical (e.g. variation between studies in the underlying effects being evaluated). ${ }^{47}$ To assess statistical heterogeneity, we examined the potential presence of moderator variables, with possible clinical and methodological heterogeneity examined descriptively. Heterogeneity was assessed to aid interpretation of the meta-analytic findings, as without knowing how consistent the results of studies are, it is not possible to determine the generalisability of the results. ${ }^{47}$

We conducted moderator analyses on the PMIEs and PTSD, depression and suicide ideation analyses, including variables where there were at least three studies in each subcategory. ${ }^{48}$ We individually examined the following variables as potential moderators of the association between PMIEs and mental health: participant age, whether the transgressive act was experienced in a military or non-military context, participant gender, study location (USA versus other) and whether or not the study utilised a measurement tool that solely examined exposure to PMIEs or if a tool was used which conflated PMIE exposure with the impact of effects (discussed in the following section). These moderators were chosen for the present analysis as sufficient data $(k \geq 3)$ was available to examine their impact on the effects. Meta-regression was used when a moderator was a continuous variable (e.g. participant age) to quantify the relationship between the magnitude of the moderator and the PMIEs (the mental health disorder effect). ${ }^{47}$

Publication bias of the relationship between PMIEs and each mental health disorder analyses was examined by creating funnel plots to provide a visual representation of the data. Rank correlation tests $^{49}$ and regression tests ${ }^{50}$ were conducted to determine if there was any evidence of publication bias. Duval and Tweedie's trim and fill procedure was also used to examine the presence of potential publication bias. $^{51}$

\section{Study sample}

Twelve of the 13 studies identified were cross-sectional, with one notable exception. ${ }^{7}$ Studies were published between 2011 and 2017 and involved a total of 6373 participants (range of 60-2095). Most participants were male, with an age range of 22.0-64.0 years. The majority of studies examined PMIEs in military samples in relation to military deployment (e.g. feeling troubled by having witnessed others' immoral acts while on deployment, ${ }^{7}$ see Table 1). In non-military samples, exposure to moral and/or ethical dilemmas in the workplace were investigated. Studies in non-military samples examined exposure to PMIEs in journalists who covered the 2011 Norway terror attack (e.g. work description included tasks that went against personal values), ${ }^{8}$ teachers exposed to community violence in El Salvador (e.g. witnessing actions by other school staff that led to the suffering of students), ${ }^{17}$ veterinarians who experienced morally significant events during veterinary practice (e.g. performed euthanasia for reasons they do not agree with ${ }^{16}$ ) and police officers who killed or caused serious injury in the line of duty. ${ }^{9}$ Non-validated assessments of workplace PMIEs were used by six studies (see Table 1), with many informed either by theory or previous research of moral injury, ${ }^{17,22}$ interviews with participants ${ }^{19}$ or via focus groups. ${ }^{8,16,20}$ Three included studies utilised validated measures of occupation-related trauma exposure as a proxy measure for PMIEs exposure. ${ }^{9,21,18}$ Four studies ${ }^{4,5,7,14}$ used the Moral Injury Events Scale (MIES) ${ }^{7}$ or the Moral Injury Questionnaire-Military Version, ${ }^{14}$ which assess exposure to both PMIEs (e.g. 'I saw things that were morally wrong') as well as emotional outcomes (e.g. 'I am troubled by having witnessed others' immoral acts') on the same items. This may confound exposure to PMIEs with the effects of exposure and could affect the reported effect sizes. ${ }^{2}$ Time since PMIEs was often unreported, with a few studies either stating that the participants were still in active military/police service or with the PMIE related to service in the Vietnam War. ${ }^{18}$

\section{Results}

\section{PTSD}

Twelve studies assessed the relationship between PMIEs and PTSD with a variety of measures, of which ten reported significant findings (see Table 1). Most studies assessed PTSD symptoms with the Posttraumatic Stress Disorder Checklist ${ }^{52}$; however, no marked differences in the PMIEs-PTSD association by PTSD measurement tool were observed. For the PMIEs and PTSD association, the weighted mean effect size was $0.30(P<0.0001,95 \%$ CI 0.20 0.39 ). This effect size is statistically significant and meets criteria for a moderate effect, suggesting that PMIEs account for approximately $9.4 \%$ of the variance in PTSD. The effect sizes of PMIEs and PTSD ranged between 0.02 and 0.65 , with some of the largest effects found in military samples (see Table 2). A potential outlier was Ferrajão and Oliveira, ${ }^{19}$ although a small positive relationship between PMIEs and PTSD was found, no significant differences in PTSD symptoms were found between those who did and those who did not report exposure to PMIEs. A non-significant positive association between PMIEs and PTSD was also observed by Bryan et al., ${ }^{4}$ however this effect was small (effect size of 0.02).

\begin{tabular}{|c|c|c|c|c|}
\hline \multirow[b]{2}{*}{ Study } & \multicolumn{4}{|c|}{ Effect size, Pearson's $r$} \\
\hline & PTSD & Depression & Suicidality & Anxiety \\
\hline Backholm and Idas ${ }^{8}$ & $0.36^{* \star *}$ & & & \\
\hline Bryan et al. ${ }^{4}$ & 0.02 & -0.05 & 0.13 & \\
\hline Bryan et al. ${ }^{5}$ & $0.22 * * *$ & $0.20 * * *$ & & $0.16^{* * *}$ \\
\hline Crane et al. ${ }^{16}$ & & & & $0.18^{* * *}$ \\
\hline Currier et al. ${ }^{14}$ & $0.65^{* * *}$ & $0.39 * * *$ & $0.14^{*}$ & \\
\hline Currier et al. ${ }^{17}$ & $0.26 * * *$ & & & \\
\hline Dennis et al. ${ }^{18}$ & $0.33^{* * *}$ & $0.27^{* * *}$ & $0.13^{* *}$ & \\
\hline Ferrajão and Oliveira ${ }^{19}$ & 0.23 & 0.03 & & \\
\hline Komarovskaya et al. ${ }^{9}$ & $0.28 * * *$ & & & \\
\hline Nash et al. ${ }^{7}$ & $0.28 * * *$ & $0.40 * * *$ & & $0.28^{* * *}$ \\
\hline Ritov and Barnetz ${ }^{20}$ & $0.24^{* * *}$ & & & \\
\hline Tripp et $a .^{21}$ & $0.42^{* * *}$ & 0.19 & $0.27 *$ & \\
\hline Wilk et al. ${ }^{22}$ & $0.18^{* * *}$ & & & \\
\hline
\end{tabular}


Heterogeneity analysis was significant $(Q(11)=90.4 ; P<0.0001$; $\left.I^{2}=92.01 \%\right)$, and potential moderating variables were examined to determine whether study characteristics accounted for differences in the results. ${ }^{47}$ Between-group differences in effect size related to study-level moderators were examined by the between-group $Q$ statistic within a random effects model. Results revealed no significant moderator effect on the association between PMIEs and PTSD of participant age (between-group $Q(1)=0.14 ; P=0.71$ ), percentage of male participants in the study $(Q(1)=0.23 ; P=0.62)$, whether the PMIE was military versus non-military related $(Q(1)=0.003 ; P=$ 0.95), whether the measurement of PMIEs conflated event exposure with the emotional effects of exposure $(Q(1)=0.08 ; P=0.78)$ or study location (USA versus other, $Q(1)=0.06 ; P=0.80$ ).

No evidence for publication bias was found for the PMIEs and PTSD analysis. Visual inspection, rank correlation $(P=0.84)$ and Egger's tests $(P=0.72)$ indicated non-asymmetric funnel plots. Furthermore, the trim and fill procedure did not suggest the imputation of any studies for this analysis, indicating a lack of publication bias.

\section{Depression}

Seven studies assessed the relationship between PMIEs and depression, four of which reported significant findings. Studies largely used the Beck Depression Inventory, Second Edition ${ }^{53}$ or The Patient Health Questionnaire- $9^{54}$ to assess depression. Pearson's $r$ effect sizes for the association between PMIEs and depression ranged between -0.05 and 0.40 . No marked differences in the PMIEs-depression association were observed based on the depression measure used, although Ferrajão and Oliveira ${ }^{19}$ was the only study to examine depression with the depression subscale of the Brief Symptom Inventory ${ }^{55}$ and found a particularly small association between PMIE and depression (effect size of 0.03). All studies examining the relationship between PMIEs and depression were conducted with military samples, with the majority conducted in the USA $(k=6)$. The mean effect size of the PMIEs and depression association was 0.23 , meeting criteria for a small effect, and was statistically significant $(P=0.0002 ; 95 \%$ CI $0.11-0.37)$. This indicates that PMIEs accounted for $5.2 \%$ of the variance in depression. Notably, Bryan et al. ${ }^{4}$ found a negative association between PMIEs and depression (overall effect size of -0.05), meaning that PMIE was associated with fewer depression symptoms, although the strength and nature of the PMIEs-depression relationship varied by event type (see Supplementary Table 3).

The results of the heterogeneity analysis were significant $(Q(6)$ =39.56; $\left.P<0.0001 ; I^{2}=88.93\right)$. No significant study moderators were found (participant age, $Q(1)=1.39, P=0.23$; percentage of male participants, $Q(1)=1.88, P=0.17$; whether the measurement of PMIE conflated event exposure with the emotional effect of exposure, $Q(1)=0.23 ; P=0.63)$.

No evidence of publication bias was found. The rank correlation $(P=0.77)$ and Egger's $(P=0.18)$ tests were not significant and the trim and fill procedure did not recommend the imputation of any additional studies for this analysis, indicative of a lack of publication bias.

\section{Suicidality}

Four studies assessed the PMIEs-suicidality association, three of which reported significant findings. All studies were based in the USA and examined PMIEs in a military context. Meta-analysis examining PMIEs and suicidality identified a small, significant mean effect size of $0.14(P<0.0001 ; 95 \%$ CI $0.08-0.20)$. This mean effect size meets the criteria for a small effect, suggesting that PMIEs is associated with approximately $2.0 \%$ of the variance in suicidality. Studies reporting on the relationship between
PMIEs and suicidality all utilised military samples, with effect sizes ranging from 0.13 to 0.27 .

The results of the heterogeneity analysis were non-significant $\left(Q(3)=1.27 ; \quad P=0.74 ; I^{2}=0.00\right)$. Given the small number of included studies, a non-significant result cannot necessarily be interpreted as evidence of no statistical heterogeneity as the test may lack power to detect significant heterogeneity when present. ${ }^{47}$ Thus, between-group differences in effect size related to study-level moderators were examined by a random effects model to ensure the PMIEs-suicidality association was thoroughly explored. No significant moderators of effect size were found (age, $Q(1)=0.18 ; P=0.67$; percentage of male participants, $Q(1)=0.01$; $P=0.94)$. Other moderators, such as study location, whether the tool used to measure PMIE conflated PMIE exposure with the emotional effects of exposure, and PMIE type (e.g. military versus nonmilitary) could not be examined owing to insufficient data for a meaningful contrast between subgroups. Only one study used a non-validated measure of PMIEs, ${ }^{14}$ and it reported findings (effect size of 0.14) that were not inconsistent with other studies that utilised validated measures of PMIE (e.g. Bryan et al. ${ }^{4}$ and Dennis et al. ${ }^{18}$ ). No evidence for publication bias was found for the PMIEs and suicidality analysis. Visual inspection, rank correlation $(P=0.33)$ and Egger's tests $(P=0.38)$ suggest non-asymmetric funnel plots. The trim and fill procedure did not recommend the addition of any further studies for this analysis, suggesting a lack of publication bias.

\section{Anxiety}

Three studies examined the association between PMIEs and anxiety, thus it was not possible to utilise meta-analytic methods. ${ }^{5,7,16}$ One study examined the relationship between PMIEs and anxiety in veterinarians, ${ }^{16}$ whereas Bryan et al. ${ }^{5}$ and Nash et al. ${ }^{7}$ examined military-related exposure to PMIEs. PMIEs were significantly associated with anxiety symptoms across all three studies (range of $0.16-$ 0.28 , see Table 2). The relationship between PMIEs and anxiety was fairly small in the non-military sample, ${ }^{16}$ which may reflect the nature and/or intensity of the PMIEs experienced. Bryan et al. ${ }^{5}$ found all event types (e.g. transgressions - other, transgressions self, betrayal) to be significantly positively associated with anxiety, with the strongest relationship found between anxiety and perceived betrayal (effect size of 0.219 ; see Supplementary Table 3 ).

\section{Hostility}

Three studies examined the relationship between hostility and PMIEs, all in a military context. ${ }^{5,18,22}$ In all studies, PMIEs and exposure to wartime atrocities (e.g. acting in ways that violate one's moral code; hurting, killing or mutilating bodies of civilians and enemy combatants) was positively and significantly associated with hostile behaviour, although some effects were small (Bryan et al. ${ }^{5}$ effect size of 0.21; Dennis et al. ${ }^{18}$ effect size of 0.18). ${ }^{44}$ The larger effect reported by Wilk and colleagues ${ }^{22}$ (effect size of 0.41 ) may reflect the fact that the sample participated in the study during deployment to Iraq in 2007, a non-validated measure of PMIEs was used and the non-validated measure of hostility largely focused on aggression towards other unit members (e.g. 'In the past month, have you threatened a unit member with physical violence?').

\section{Resilience, social adjustment and positive affect}

The relationship between PMIEs and psychological resilience, or the ability to recover from stressor events in the past 4 weeks, was examined by Crane et al. ${ }^{16}$ with the Brief Resilience Scale, ${ }^{56}$ with a significant negative association found between PMIEs and resilience 


\begin{tabular}{|c|c|c|c|c|c|}
\hline \multirow[b]{2}{*}{ Study } & \multicolumn{5}{|c|}{ Effect size, Pearson's $r$} \\
\hline & Social adjustment & Positive affect & Stress & Hostility & Resilience \\
\hline Crane et al. ${ }^{16}$ & & & $0.24^{* * *}$ & & $-0.17 * * *$ \\
\hline Dennis et al..$^{18}$ & & & & $0.18^{* * *}$ & \\
\hline Bryan et al. ${ }^{5}$ & & & & $0.21 * * *$ & \\
\hline Ferrajão and Oliveira $^{19}$ & -0.03 & & & & \\
\hline Nash et al. ${ }^{7}$ & $-0.29 * * *$ & $-0.15^{\star \star *}$ & & & \\
\hline Wilk et al. ${ }^{22}$ & & & & $0.41 * * *$ & \\
\hline
\end{tabular}

(effect size of -0.17; Table 3). Consistent with this, Crane et al. ${ }^{16}$ also found a positive association between PMIEs and self-reported symptoms of stress (effect size of 0.24).

Nash et al. $^{7}$ examined the relationship between military related PMIEs and positive affect and social adjustment. PMIEs were significantly negatively associated with positive affect (effect size of -0.15 ) and social adjustment (effect size of -0.29 ), indicating that higher levels of PMIEs were associated with less self-reported social support and less positive affect. ${ }^{7}$ In keeping with these findings, Ferrajão and Oliveira ${ }^{19}$ also found a small, but not statistically significant negative relationship between perceived social support and PMIEs (effect size of -0.03). However, these findings should be interpreted cautiously as Nash et l. $^{7}$ used the MIES, which confounds PMIE exposure with outomes, ${ }^{3}$ whereas Ferrajão and Oliveira ${ }^{19}$ used a non-validated PMIE measure.

\section{Discussion}

The aim of this review was to examine the relationship between exposure to PMIEs incurred as a result of occupation and mental health outcomes. Although based on a relatively small number of articles, the results indicate that a small-to-moderate relationship between PMIEs and PTSD and depression is evident, although the associations with other mental health symptomology appears less certain.

The strongest relationship was found between PMIEs and PTSD, consistent with previous studies that report that the common symptoms of moral injury are intrusive thoughts, intense negative appraisals (e.g. shame, guilt, disgust, etc.) and reliance on cognitive avoidance as a (maladaptive) coping strategy. ${ }^{1}$ The experience of such PTSD symptoms has also been found cross-culturally in qualitative studies of moral injury in war veterans in Zimbabwe, ${ }^{57}$ where pastoral care was experienced as particularly efficacious in managing intrusive thoughts and negative affect. Although study location (USA versus Other) was not found to be a significant moderator in this analysis, included studies were largely conducted in Western environments. Additional investigation of the experiences and impact of occupation-related PMIEs in non-Western contexts would be useful to further the understanding of cross-cultural differences and similarities in mental health outcomes following PMIEs and how best to support morally injured individuals.

A statistically significant, although small relationship between depression and PMIEs was found in military personnel; however, civilian data on this association was lacking. Characteristic symptoms of depression include social withdrawal, self-depreciating emotions and a loss of meaning, ${ }^{58}$ all of which have been reported in qualitative studies following military-related moral injury. ${ }^{6}$ Similar symptoms of depression and psychological distress have also been reported in qualitative studies of humanitarian aid workers who experience work-related moral challenges (e.g. a lack of resources meaning they cannot provide adequate healthcare to all patients ${ }^{59,60}$ ).

Suicidality was significantly associated with PMIEs in military personnel with a small effect. However, this relationship may be less reliable as only three studies report significant findings. Alternatively, it is possible that the relationship between suicidality PMIEs may be an indirect effect caused by other associated risk factors or consequences of PMIEs, such as depression or PTSD, ${ }^{4,61,62}$ and warrants further research.

A modest relationship between PMIEs, anxiety, hostility, poor resilience and less social support was also examined in this review. The relationship between PMIEs and hostility is in keeping with recent research of military-related PMIEs causing anger or hostility that persists for several years post-deployment, even after controlling for PTSD symptoms. ${ }^{63}$ Nonetheless, additional investigation is required to explore the PMIEs-hostility relationship in non-military contexts.

Taken together, the results suggest a negative impact of PMIEs on psychological adjustment, in both a military and non-military occupational context. However, PMIEs only accounted for a modest proportion of the variance in PTSD, depression and suicidality. It may be valuable for future studies to consider other risk factors and instrumental moderator variables for such psychological adjustment difficulties. Given the lack of a widespread, substantial impact on mental health, it also may be of interest to consider whether exposure to PMIEs might be linked to other outcomes both in terms of practical (e.g. resigning from one's work) or positive change (e.g. post-traumatic growth).

\section{Strengths and limitations}

The results of this study must be considered in light of the limitations. First, most included studies examined PMIEs in a military context $(k=10)$. Other occupational groups, including firefighters, relief aid workers and social workers, are exposed to traumatic and PMIEs and additional research is needed to fully understand the impact of such stressors on their mental health and wellbeing. Second, all studies measured exposure to PMIEs by selfreport measures, many of which were not validated. .,16,17,19,20 $^{2}$ Several studies also used measures of PMIEs that have methodological issues ${ }^{2}$ (e.g. confounding exposure to transgressive events with exposure effects ${ }^{4,5,7,14}$ ), although this was not found to be a significant moderator for the PTSD and depression analyses. In some cases, a proxy measure of PMIEs, such as exposure to war-time atrocities, ${ }^{18}$ was used which highlights the lack of consistency in the literature of the types of events that can cause moral injury. Nonetheless, to further our understanding of the impact of PMIEs on mental health, a valid and reliable assessment of PMIEs and moral injury outcomes is required. Third, this review was not preregistered on PROSPERO or a similar register. Finally, the majority of studies included in this review examined PMIEs in a US or Western context (e.g. Norway, Australia), with a few notable 
exceptions (e.g. Israel, El Salvador) and additional research in nonWestern, low- or middle-income countries is needed.

\section{Clinical implications}

Our findings indicate that occupational PMIEs can potentially have an, albeit small, impact on the mental health of both military and civilian personnel. Importantly, this suggests that moral injury is not a concept that is only relevant within a military context and can potentially be experienced in other occupational settings, although additional research in non-military samples is recommended to more fully understand this experience. What evidence there is suggests that individuals who experience PMIE may be at risk of PTSD and depressive disorders. Previous reviews suggest that some treatment approaches for these disorders may be insufficient in cases of moral injury. ${ }^{6}$ Treatment for PTSD, for example, may not adequately address all negative sequelae present in those with moral injury. Future research exploring the impact of PMIEs on psychopathology over time, as well as randomised control trials directly evaluating treatment approaches following PMIEs would be beneficial.

\section{Directions for future research}

This review suggests a number of additional areas for exploration that may prove beneficial for our understanding of moral injury. Although the evidence regarding the mental health outcomes of PMIEs appears to be at most modest, what seems particularly clear is that there is a lack of high-quality evidence published on this topic. This, in part, may reflect the fact that moral injury is a relatively emerging concept $t^{1,2}$ and there is a need for considerably more research, including the design and validation of assessments that measure the impact of PMIE exposure as well as the outcomes of moral injury. As it stands, some existing measures do not include exposure to a variety of PMIEs or confound PMIE exposure with the psychological effects of exposure. ${ }^{2,3}$ The development of highquality measurement tools would allow for reliable investigations into the existence and prevalence of moral injury in both military and non-military environments and would further our theoretical understanding of whether moral injury is a distinct concept. This line of research could also aid in exploring whether there are particular experiences that are more likely to cause moral injury, as well as the precursors and the factors associated with vulnerability or resilience following moral injury. As not all individuals who experience trauma necessarily develop PTSD, exposure to PMIEs may similarly not always result in moral injury and additional research is needed to better understand PMIE outcomes. For example, the pernicious effects of moral injury may depend on one's appraisal of the transgressive act and the coping strategies used.

In the wider literature, previous studies in healthcare professionals have found years of occupation experience to be significantly positively associated with moral distress, contributing to staff burnout and resignation..$^{10,11}$ Although moral distress differs from moral injury in that the conditions in which it can be experienced are often more limited (e.g. healthcare professionals are prevented from acting on their judgement of the right thing to do largely by institutional restraints, such as pressure to minimise costs $\left.{ }^{64}\right)$; nonetheless, it is possible that factors contributing to poor mental health outcomes following moral distress may be applicable in cases of moral injury and should be pursued further.

Although only examined by two studies, exposure to specific PMIEs (e.g. transgressions - other, transgressions - self, betrayal) were differentially associated with mental health ${ }^{4,5}$ (Supplementary Table 3). One study ${ }^{5}$ found a particularly strong relationship between perceived betrayal and mental health difficulties. As this sample had very recently returned from deployment to
Afghanistan, this type of PMIE could be more salient to participants when responding to study measures. This highlights the need for moral injury to be examined as a function of PMIE type and time since event to better understand moral injury.

\section{Summary}

This review presents a comprehensive review and meta-analysis of the relationship between exposure to occupational-related PMIEs and mental health in both military and non-military connected personnel. We found small yet significant associations between PMIEs and PTSD and depression. A less reliable relationship between PMIEs, anxiety, hostility and suicidality was also observed. Given the limited number of high-quality studies available, only tentative conclusions about the association between PMIEs and mental health disorders can be made at this stage. This study highlights that considerably more research is needed in the field of moral injury, including the development of valid assessments of the impact of PMIEs exposure and outcomes. We suggest that additional investigations, particularly in relevant non-military connected populations, and other influential moderators and outcomes are considered in future research into moral injury.

Victoria Williamson, PhD, Sharon A.M. Stevelink, PhD, Neil Greenberg, MD, King's Centre for Military Health Research, King's College London, UK.

Correspondence: Victoria Williamson, Kings Centre for Military Health Research, Institute of Psychology, Psychiatry and Neuroscience, King's College London, London SE5 9RJ, UK. Email: victoria.williamson@kcl.ac.uk

First received 25 Oct 2017, final revision 6 Feb 2018, accepted 23 Feb 2018

\section{Supplementary material}

Supplementary material is available online at https://doi.org/10.1192/bjp.2018.55.

\section{References}

1 Litz BT, Stein N, Delaney E, Lebowitz L, Nash WP, Silva C, et al. Moral injury and moral repair in war veterans: a preliminary model and intervention strategy. Clin Psychol Rev 2009; 29: 695-706.

2 Farnsworth JK, Drescher KD, Nieuwsma JA, Walser RB, Currier JM. The role of moral emotions in military trauma: implications for the study and treatment of moral injury. Rev Gen Psychol 2014; 18(4): 249. doi:10.1037/gpr0000018.

3 Frankfurt S, Frazier P. A review of research on moral injury in combat veterans. Mil Psychol 2016; 28: 318-30.

4 Bryan AO, Bryan CJ, Morrow CE, Etienne N, Ray-Sannerud B. Moral injury, suicidal ideation, and suicide attempts in a military sample. Traumatology (Tallahass Fla) 2014; 20: 154-60.

5 Bryan CJ, Bryan AO, Anestis MD, Anestis JC, Green BA, Etienne N, et al. Measuring moral injury. Assessment 2016; 23: 557-70.

6 Drescher KD, Foy DW, Kelly C, Leshner A, Schutz K, Litz B. An exploration of the viability and usefulness of the construct of moral injury in war veterans. Traumatology (Tallahass Fla) 2011; 17: 8-13.

7 Nash WP, Marino Carper TL, Mills MA, Au T, Goldsmith A, Litz BT. Psychometric evaluation of the moral injury events scale. Mil Med 2013; 178: 646-52.

8 Backholm K, Idås T. Ethical dilemmas, work-related guilt, and posttraumatic stress reactions of news journalists covering the terror attack in Norway in 2011. J Trauma Stress 2015; 28: 142-8.

9 Komarovskaya I, Maguen S, McCaslin SE, Metzler TJ, Madan A, Brown AD, et al. The impact of killing and injuring others on mental health symptoms among police officers. J Psychiatr Res 2011; 45: 1332-6.

10 Lamiani G, Borghi L, Argentero P. When healthcare professionals cannot do the right thing: a systematic review of moral distress and its correlates. $J$ Health Psychol 2017; 22: 51-67.

11 Oh Y, Gastmans C. Moral distress experienced by nurses. Nurs Ethics 2015; 22: 15-31.

12 Dombo EA, Gray C, Early BP. The trauma of moral injury: beyond the battlefield. J Relig Spiritual Soc Work Soc Thought 2013; 32: 197-210. 
13 Moher D, Liberati A, Tetzlaff J, Altman DG, Grp P. Preferred reporting items for systematic reviews and meta-analyses: the PRISMA statement (Reprinted from annals of internal medicine). Phys Ther 2009; 89: 873-80.

14 Currier JM, Holland JM, Drescher K, Foy D. Initial psychometric evaluation of the moral injury questionnaire-military version. Clin Psychol Psychother 2015; 22: 54-63.

15 Ajetunmobi O. Making Sense of Critical Appraisal. Arnold, 2002.

16 Crane M, Phillips J, Karin E. Trait perfectionism strengthens the negative effects of moral stressors occurring in veterinary practice. Aust Vet J 2015; 93: 354-60.

17 Currier JM, Holland JM, Rojas-Flores L, Herrera S, Foy D. Morally injurious experiences and meaning in Salvadorian teachers exposed to violence. Psychol Trauma Theory, Res Pract Policy 2015; 7: 24-33.

18 Dennis PA, Dennis NM, Van Voorhees EE, Calhoun PS, Dennis MF, Beckham JC Moral transgression during the Vietnam War: a path analysis of the psychological impact of veterans' involvement in wartime atrocities. Anxiety, Stress Coping 2017; 30: 188-201

19 Ferrajão PC, Oliveira RA. Self-awareness of mental states, self-integration of personal schemas, perceived social support, posttraumatic and depression levels, and moral injury: a mixed-method study among Portuguese war veterans. Traumatology (Tallahass Fla) 2014; 20: 277-85.

20 Ritov G, Barnetz Z. The interrelationships between moral attitudes, posttraumatic stress disorder symptoms and mixed lateral preference in Israel reserve combat troops. Int J Soc Psychiatry 2014; 60(6): 606-12. doi:10.1177/ 0020764013502469

21 Tripp JC, McDevitt-Murphy ME, Henschel AV. Firing a weapon and killing in combat are associated with suicidal ideation in OEF/OIF veterans. Psycho Trauma Theory, Res Pract Policy 2016; 8: 626-33.

22 Wilk JE, Bliese PD, Thomas JL, Wood MD, McGurk D, Castro CA, et al. Unethical battlefield conduct reported by soldiers serving in the Iraq War. J Nerv Ment Dis 2013; 201: 259-65.

23 Beck AT, Steer RA. Manual for the beck anxiety inventory. Psychological Corporation, 1990

24 Dozois DJA, Covin R. The Beck Depression Inventory-II (BDI-II), Beck Hopelessness Scale (BHS), and Beck Scale for Suicide Ideation (BSS). In Comprehensive Handbook of Psychological Assessment Volume 2, Personality Assessment (eds. MJ Hilsenroth, DL Segal): 50-69. John Wiley \& Sons, 2004.

25 Blake DD, Weathers FW, Nagy LM, Kaloupek DG, Gusman FD, Charney DS, et al. The development of a clinician-administered PTSD Scale. J Trauma Stress 1995 8(1): 75-90. doi:10.1007/BF02105408.

26 Weiss DS, Brunet A, Best SR, Metzler TJ, Liberman A, Marmar CR. Frequency and severity approaches to indexing exposure to trauma: the critical inciden history questionnaire for police officers. JOTS 2010; 23(6): 734-43. doi:10.1002/ jts.20576.

27 Han K, Weed NC, Calhoun RF, Butcher JN. Psychometric characteristics of the MMPI-2 Cook-Medley Hostility scale. J Pers Assess 1995; 65(3): 567-85. doi:10.1207/s15327752jpa6503_15.

28 Lovibond S, Lovibond P. Depression anxiety and stress scale. Manual for the Depression and Anxiety Stress Scales. DASS Publications, 1995

29 King LA, King DW, Vogt DS, Knight J, Samper RE. Deployment Risk and Resilience Inventory: a collection of measures for studying deployment-related experiences of military personnel and veterans. Mil Psych 2006; 18(2): 89-120. doi:10.1207/s15327876mp1802_1.

30 Spitzer RL, Kroenke JP, Williams L. A brief measure for assessing generalized anxiety disorder: the GAD-7. Arch Intern Med 2006; 166(10): 1092-7. doi:10.1001/archinte.166.10.1092

31 Horowitz L, Wilner M, Alvarez N, Weiss W, Marmar DS, Neal CR, et al. Impact of event scale - revised. Psychosom Med 1979; 41: 209-18.

32 Cohen S, Hoberman H. Interpersonal support evaluation list (ISEL). J App/ SOC Psychol 1983; 58: 304-9. doi:10.1017/CBO9781107415324.004

33 King LA, King DW, Leskin G, Foy DW. The Los Angeles symptom checklist: a selfreport measure of posttraumatic stress disorder. Assessment 1995; 2(1): 1-17.

34 Lauterbach D, Vrana S, King DW, King LA. Psychometric properties of the civilian version of the Mississippi PTSD Scale. J Trauma Stress 1997; 10(3): 499-513. doi:10.1023/A:1024801607043.

35 Watson D, Clark LA, Tellegen A. Development and validation of brief measures of positive and negative affect: the PANAS scales. J Pers Soc Psychol 1988; 54(6): 1063

36 Gameroff MJ, Wickramaratne P, Weissman MM. Testing the Short and Screener versions of the Social Adjustment Scale - Self-report (SAS-SR). Int J Methods Psychiatr Res 2012; 21(1): 52-65. doi:10.1002/mpr.358.
37 Linehan MM, Clinics T. Suicidal behaviors questionnaire. Assessment 2001; 8(4): 443-54.

38 Beck AT, Kovacs M, Weissman A. Assessment of suicidal intention: the scale for suicide ideation. J Consult Clin Psychol 1979; 47(2): 343. doi:10.1037/0022$006 \times .47 .2 .343$

39 Spielberger CD. State-trait anger expression inventory. J Am Coll Cardiol 1988 36: 1781-8. doi:10.1177/0306624X9804200206

40 Wilson JP, Zigelbaum SD. The Vietnam veteran on walk: The relation of posttraumatic stress disorder to criminal behaviour. Behav Sci Law 1983; 1(3): 69-83. doi:10.1002/bsl.2370010308.

41 Viechtbauer W. Conducting meta-analyses in R with the meta for package. J Stat Softw 2010; 6(3): 1-48. doi:10.1103/PhysRevB.91.121108.

42 Field AP. Meta-analysis of correlation coefficients: a Monte Carlo comparison of fixed- and random-effects methods. Psychol Methods 2001; 6: 161-80.

43 Rosenthal R. Writing meta-analytic reviews. Psychol Bull 1995; 118: 183-92.

44 Cohen J. Statistical Power Analysis for the Behavioral Sciences. Elsevier, 1988.

45 Hedges LV, Vevea JL. Fixed- and random-effects models in meta-analysis. Psychol Methods 1998; 3: 486-504.

46 Huedo-Medina TB, Sánchez-Meca J, Marín-Martínez F, Botella J. Assessing heterogeneity in meta-analysis: Q statistic or $\mathrm{I}^{2}$ index? Psychol Methods 2006; 11: 193-206.

47 Borenstein M, Hedges LV, Higgins JPT, Rothstein HR. Introduction to MetaAnalysis. John Wiley \& Sons, 2011.

48 Bakermans-Kranenburg MJ, van IJzendoorn MH, Juffer F. Less is more: metaanalyses of sensitivity and attachment interventions in early childhood. Psycho Bull 2003; 129: 195-215.

49 Begg CB, Mazumdar M. Operating characteristics of a rank correlation test for publication bias. Biometrics 1994; 50: 1088.

50 Egger M, Smith GD, Schneider M, Minder C. Bias in meta-analysis detected by a simple, graphical test. BMJ 1997; 315: 629-34.

51 Duval S, Tweedie R. Trim and fill: a simple funnel-plot-based method of testing and adjusting for publication bias in meta-analysis. Biometrics 2000; 56: 455-63.

52 Weathers FW, Litz BT, Herman DS, Huska JA, Keane TM. The PTSD Checklist (PCL): Reliability, Validity, and Diagnostic Utility. Paper presented at the Annual Convention of the International Society for Traumatic Stress Studies, San Antonio, TX, October, 1993.

53 Beck A, Steer R, Brown G. Beck Depression Inventory-II (BDI-II). The Psychological Corporation, 1993.

54 Kroenke K, Spitzer RL, Williams JBW. The PHQ-9. J Gen Intern Med 2001; 16 606-13.

55 Derogatis LR. BSI Brief Symptom Inventory: Administration, Scoring, and Procedure Manual (4th edn). National Computer Systems, 1993.

56 Smith BW, Dalen J, Wiggins K, Tooley E, Christopher P, Bernard J. The brief resilience scale: assessing the ability to bounce back. Int J Behav Med 2008; 15(3): 194-200. doi:10.1080/10705500802222972.

$57 \mathrm{Moyo} \mathrm{H}$. Pastoral care in the healing of moral injury: a case of the Zimbabwe National Liberation War Veterans. HTS Teol Stud/Theol Stud 2015; 71: 1-11.

58 American Psychiatric Association. Diagnostic and Statistical Manual of Mental Disorders, (5th edn). American Psychiatric Association, 2013.

59 Hunt MR. Ethics beyond borders: how health professionals experience ethics in humanitarian assistance and development work. Dev World Bioeth 2008; 8: 59-69.

60 Schwartz L, Sinding C, Hunt M, Elit L, Redwood-Campbell L, Adelson N, et al. Ethics in humanitarian aid work: learning from the narratives of humanitarian health workers. AJOB Prim Res 2010; 1: 45-54.

61 Griffith J. Suicide and war: the mediating effects of negative mood, posttraumatic stress disorder symptoms, and social support among Army National Guard soldiers. Suicide Life Threat Behav 2012; 42: 453-69.

62 LeardMann CA, Powell TM, Smith TC, Bell MR, Smith B, Boyko EJ, et al. Risk factors associated with suicide in current and former US military personnel. JAMA 2013; 310(5): 496-506. doi:10.1001/jama.2013.65164.

63 Worthen M, Ahern J. The causes, course, and consequences of anger problems in veterans returning to civilian life. J Loss Trauma 2014; 19: 355-63.

64 Epstein EG, Delgado S. Understanding and addressing moral distress. Online J Issues Nurs 2010; 15(3). doi:10.3912/0JIN.Vol15N003Man01. 